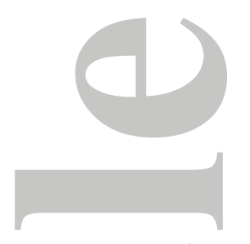

\title{
Endurance exercise: An important therapeutic adjuvant in the overall treatment of myositis?
}

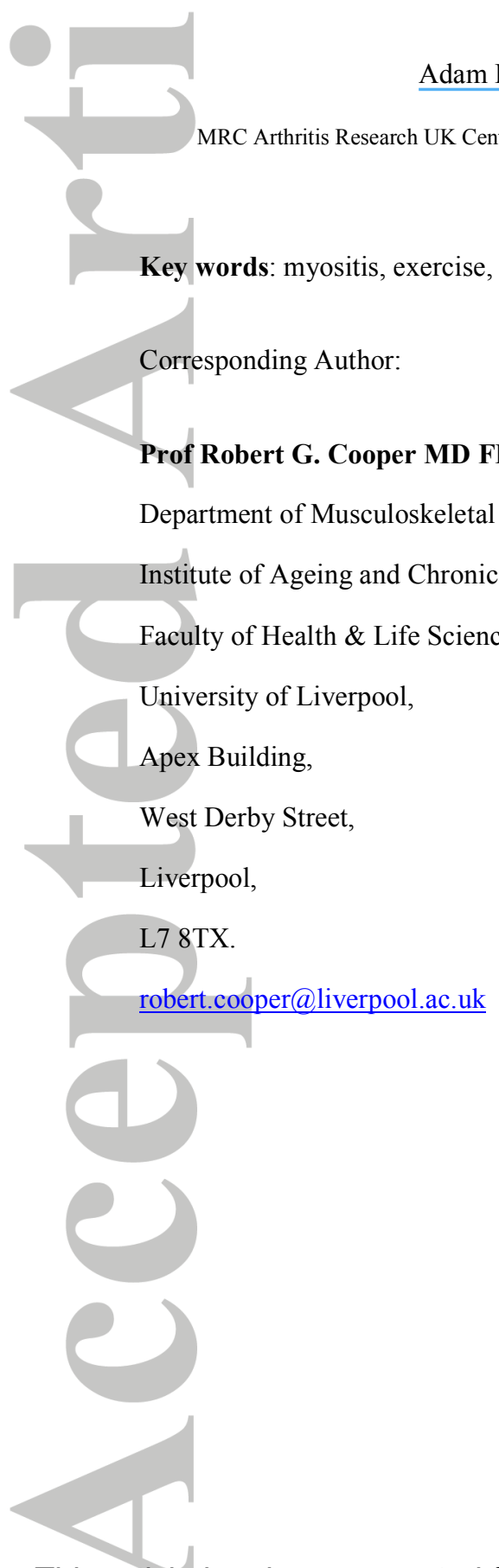

Adam P. Lightfoot, PhD, Robert G. Cooper, MD, FRCP

This article has been accepted for publication and undergone full peer review but has not been through the copyediting, typesetting, pagination and proofreading process which may lead to differences between this version and the Version of Record. Please cite this article as an 'Accepted Article', doi: 10.1002/art.39615 (C) 2016 American College of Rheumatology Received: Jan 21, 2015; Accepted: Jan 26, 2016 


\section{Introduction}

Idiopathic inflammatory myopathy (myositis) is an autoimmune disorder associated with myositis specific and associated auto-antibody production, and the histological detection of inflammatory cell infiltrates between and within skeletal muscle fibres in diagnostic muscle biopsies from affected patients. The infiltrates are associated with fibre atrophy and myonecrosis, causing weakness and disability. Given the inflammatory nature of myositis, it is no surprise that treatment currently relies on glucocorticoid use, in combination with various "disease-modifying" immunosuppressive agents. Interestingly, no one immunosuppressive regime has to-date proved superior to any other in myositis. Even with aggressive immunosuppression, muscle damage may still progress, accruing cumulative muscle atrophy and fatty replacement, which are usually irreversible. Even when immunosuppression has clearly reduced inflammatory cell loads, muscle weakness often persists; so most treated patients have detectable if stable weakness. The magnitude of inflammation detected in muscle biopsies often correlates only poorly with the degree of detected muscle weakness (1), while in an established murine model of myositis; muscle weakness appears to actually precede the infiltration of inflammatory cells (2). These discussions highlight our paucity of understanding regarding the mechanisms/s of weakness-induction in myositis. It is, however, increasingly recognised that myositis-associated muscle weakness may arise from non immune-cell mediated mechanisms, i.e. over and above that caused by immune cell infiltrations. For instance, endoplasmic reticulum (ER) stress pathway activation has received much recent attention regarding its potential role in non-inflammatory weakness induction (3). Given that immunosuppression is incompletely effective in myositis, and that non-immune cell mediated pathways are likely also involved in weakness induction, it follows that for future myositis treatments to become more effective, they must address non-inflammatory as well as inflammatory pathologies.

A key question arising in myositis is what is/are the precise cause/s of "residual" weakness, i.e. over and above that due to inflammation? Unsuppressed disease activity or irreversible damage may play a role, though both scenarios would be detectable by repeat neurophysiology (EMG), muscle biopsies and MRI scans, and subsequent treatment tailored accordingly. If retesting excludes disease activity and irreversible damage, the cause of ongoing weakness could instead be due to a myositis-induced intrinsic disturbance of intracellular bioenergetic and/or proteomic pathways, e.g. as postulated to result from chronic ER stress induction $(3,4)$. Moreover, there may be some attribution to lower-grade of muscle atrophy but which has 
not, for whatever reason, improved with the expected return towards normal activity levels following suppression of inflammation. The latter could suggest that disuse atrophy also represents a specific problem. Ongoing non-inflammatory weakness could obviously also have iatrogenic components, e.g. due to chronic glucocorticoid use. Interrogating the issue of residual weakness by the use of an exercise program has got the potential to discern between these possibilities.

\section{Exercise in myositis}

In an effort to assess non-pharmacological means of treating myositis-induced weakness, several studies have investigated the role of exercise as a clinical intervention (5). Historically, exercise in myositis patients was often advised against, based on the possibility that it may cause further damage by generating "secondary" inflammation capable of causing further muscle damage and inflammation. As if to emphasise this point, long term low dose steroid use is now a recommended treatment component in Duchenne Muscular Dystrophy, and used to minimise damage progression from just such a vicious cycle. Early exercise studies in myositis patients clearly demonstrated that it is safe for myositis patients to exercise, as muscle damage is not induced (6). Thus, exercise is deemed safe and beneficial in myositis, as well as in a wide-range of other rheumatic diseases, e.g. RA and SLE (7). In their article reported in the current issue of Arthritis and Rheumatology, Munters and colleagues have elegantly tested the efficacy of a 12-week endurance training regime in patients with established but stable myositis, and assessing a range of physiological, metabolic and inflammatory parameters, as well as assessing the effects on disease activity (8). Using state-of-the-art genomics and proteomics, Munters et al have characterised pathways directly altered in response to endurance training in myositis patients, and thus building on their previously published studies as part of a larger RCT $(9,10)$. The results from these studies demonstrate that this 12 week period of resistance exercise resulted in improved aerobic capacity, which is maintained at 1 year of follow-up. These findings appear to challenge the possibility that myositis-induced intrinsic mechanisms cause patients to remain weak, and instead suggest that simple muscle deconditioning and/or psychological factors are instead the more prominent problem. However, and given that most treated myositis patients remain weak, the existence of induced bioenergetic and/or metabolic deficits remain a possibility in some patients.

In their current article, Munters et al show significant exercise-induced beneficial changes at gene and protein component levels, as well as in immune and non-immune cell aspects of myositis pathological processes. Specifically, endurance training appears to down-regulate genes mechanistically linked to

\section{John Wiley \& Sons}

This article is protected by copyright. All rights reserved. 
myositis initiation and/or maintenance, such as $\mathrm{x}$-box binding protein (XBP) 1. This gene is known to be associated with ER stress pathway activation in myositis patients; a purported non-immune cell mediated component. The authors report simultaneous down-regulation in gene expressions related to inflammatory markers, such as CXCR7 and CD40. Additionally, gene and protein analyses in patients showed exerciseinduced beneficial changes in key components involved in muscle remodelling and mitochondrial biogenesis and function (e.g. in oxidative phosphorylation). Collectively these findings provide apparently convincing evidence that the muscle of myositis patients with established but stable disease is positively responsive to an exercise-based intervention, which is also clearly safe. The improvements demonstrated in aerobic capacity (i.e. in $\mathrm{VO}_{2}$ max) are supported by the observation of significant beneficial changes in genes and proteins associated with mitochondrial dynamics and bioenergetics. Importantly, the authors identify exercise-induced upregulation of the protective mitochondrial antioxidant enzyme peroxiredoxin 3 (PRDX3). Typically, and due to exercise-induced increases in cellular respiration, elevated production of reactive oxygen species (ROS) occurs as a bi-product of the electron transport chain (ETC). ROS are capable of causing cellular damage, by readily oxidising DNA, proteins and lipids; and in muscle are specifically thought to cause reduced force production via oxidative damage to mitochondrial components and contractile proteins (11). However, under physiological conditions any potential for damage from exerciseinduced ROS generation is contained by a network of antioxidant defence enzymes, of which PRDX3 is an essential component. The significance of the PRDX3 findings by Munters et al is that, in a pathological state (i.e. in myositis), they demonstrate that muscle mitochondria and their antioxidant defence systems are performing normally. This is in contrast to some other myopathologies, such as in the sarcopenia of ageing, where muscle is unable to mount a normal adaptive antioxidant response during exercise (12). We speculate that the elevations in the cytoprotective PRDX3 observed by Munters et al, may be a factor contributing to the overall safety and lack of exacerbation of inflammation observed in their patient cohort with exercise.

The observed beneficial changes in mitochondrial function with exercise may in-part be attributed to the expected increases in capillary density and other factors related to angiogenesis, which were likely upregulated by endurance exercise. Improving mitochondrial function may thus be a potential way by which exercise acts as a therapeutic adjuvant in myositis patients with persisting weakness in the absence of overt muscle inflammation.

In normal young men suffering tibial fractures, the expected and observed quadriceps muscle wasting is post-fracture restored to normal bulk by simple re-establishment of normal day-to-day quadriceps activity 
levels. Research has shown however that tibial fracture-related quadriceps wasting is preventable by the use of very low intensity surface electrical stimulation to just produce contractions (13), clearly suggesting that in health only very small amounts of voluntary activation may be required to maintain muscle bulk. In contrast, in patients with chronic, myositis-induced muscle atrophy, a resumption of normal day-to-day activities following suppression of disease activity clearly does not always or even usually restore muscle bulk and strength to normal. This may reflect that, in patients who have been considerably ill during the acute phase of their myositis, a degree of de-motivation regarding physical reactivation has occurred. Alternatively, a chronic overall catabolic process is induced and is ongoing in myositic muscle, even in the absence of inflammatory cells, and perhaps related to chronic ER stress pathway activation (3). Irrespective of which, both or other mechanisms contribute to chronic muscle weakness induction and atrophy in myositis, Munters et al appear to have convincingly shown that their 12 week activity program is able to overcome and reverse this tendency. Given the previous report by the authors, that strength improvements are potentially maintained 1 year post exercise training in such patients (9), a question naturally arising is whether the demonstrated beneficial genomic and proteomic changes at 12 weeks are also maintained at 1 year? Thus, longitudinal studies are now required to determine whether or not all of the beneficial changes described by Munters et al are maintained following cessation of training. It is unclear whether the proteomic and genomic changes observed by Munters et al are specific to myositis muscle, or instead representative of a normal physiological response to exercise which would be expected to be observed in normal healthy individuals. The lack of an appropriate control group limits interpretation here in this respect. However, as Munters et al suggest, the aerobic changes observed in the endurance exercise patient group, are similar to changes seen in other exercise research, for instance in similarly aged healthy individuals (14).

The mechanisms which drive and mediate the exercise-induced changes reported by Munters et al are not fully clarified here. The gene and protein changes observed may be a direct response to exercise (mechanical stimuli), or due to downstream alterations in response to factors released from muscle, such as myokines.

\section{Could myokines play a role as mediators of the beneficial effect of exercise in myositis?}

The beneficial and anti-inflammatory effects of exercise have been the topic of wide ranging research over 15-20 years. In the late 1990s evidence began to emerge that functionally important "factors" are released from muscle in response to exercise, so heralding the concept of muscle-derived cytokines (or myokines). The understanding of myokines has increased greatly in recent years, with an ever increasing 
repertoire, and indeed the whole muscle "secretome" has become a topic of considerable interest. The first myokine described following its exercise-induced release was IL-6 (15). Rheumatologists would obviously view IL-6 as a key pro-inflammatory cytokine; but IL-6 released from muscle with exercise has in fact got anti-inflammatory properties. These relate to IL-6's upregulation in response to exercise, which is independent of classic pro-inflammatory cytokines such as TNF, and instead related to $\mathrm{Ca}^{2+}$ mediated and MAPK-related mechanisms. Muscle IL-6 release with exercise is followed by elevations in circulating levels of other anti-inflammatory cytokines, including IL-10 and IL-1ra (7). Other evidence for the antiinflammatory nature of IL- 6 has been provided by the demonstration that either exercise or IL-6 infusions are capable of ameliorating endotoxin-induced inflammation (7). As well as being an anti-inflammatory mediator, muscle-derived IL-6 has also been reported to augment metabolic changes in response to exercise, by acting as an energy sensor (7). Many other cytokines are reported to be released from muscle, with several, such as IL-15, having important paracrine signalling properties. IL-15 is expressed and released from muscle in response to exercise; when it promotes anabolism and reduces adipose tissue deposition (7). Thus, there is considerable evidence to indicate that myokines likely augment the beneficial muscle adaptations seen in response to exercise. Based on this knowledge it is possible to hypothesise that the beneficial anti-inflammatory and remodelling and adaptation responses presented here by Munters et al may be mediated, at least in part, by myokines. Surprisingly, the gene and protein analyses undertaken by Munters et al did not detect changes in known or classical myokines (e.g. IL-6/IL-15). Whether or not myokines contribute to exercise-induced beneficial responses in myositis patients remains to be established.

\section{Future perspectives for exercise in myositis}

Given its demonstrable safety and efficacy, it appears appropriate and now timely to further develop the concept of using exercise as a therapeutic adjuvant in treatment strategies for myositis, though several aspects of both the impact of exercise and potential mechanisms of action will require further assessment. It seems clear that endurance exercise is a successful intervention in myositis, though the issue of intensity and duration of training will require further delineation. To address these issues, longitudinal exercise studies,

with appropriate age and activity matched healthy controls, are now needed. Such research may indicate whether fundamental beneficial changes at a cellular level are achievable by exercise, and whether these can be maintained voluntarily following cessation of specific endurance exercises. Whether low intensity stimulated contractions could be used to maintain the beneficial effects of such exercise, once achieved, also 
represents an intriguing question. Assessments of the proteins secreted from the muscles of myositis patients, and the impact of endurance exercise on them, may provide further mechanistic insights into the pathogenesis of myositis weakness-induction. Given the robust scientific technologies now available, myositis researchers appear well placed to interrogate such mechanisms and the effects of exercise upon them, and to continue to refine therapeutic methods to address the complex issue of chronic, myositisinduced muscle weakness.

\section{Author contributions}

Both authors drafted the article, revised it critically for important intellectual content and approved the submitted version.

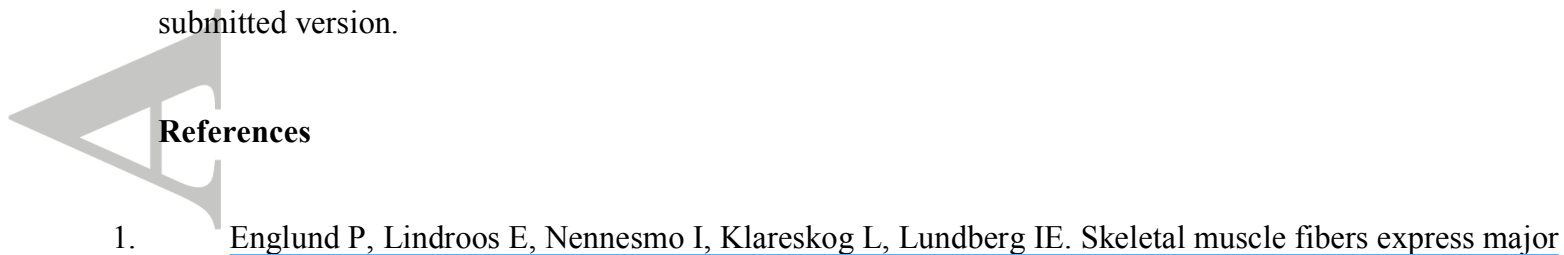
histocompatibility complex class II antigens independently of inflammatory infiltrates in inflammatory myopathies. Am J Pathol. 2001;159(4):1263-73.

2. Coley W, Rayavarapu S, Pandey GS, Sabina RL, Van der Meulen JH, Ampong B, et al. The molecular basis of skeletal muscle weakness in a mouse model of inflammatory myopathy. Arthritis Rheum. 2012;64(11):3750-9.

3. Lightfoot AP, Nagaraju, K, McArdle, A, Cooper R.G. Understanding the origin of non immune-cell mediated weakness in the idiopathic inflammatory myopathies (IIM) - Potential role of ER stress-pathways. Curr Opin Rheumatol. 2015;27(6):580-5.

4. Rayavarapu S, Coley W, Nagaraju K. An update on pathogenic mechanisms of inflammatory myopathies. Curr Opin Rheumatol. 2011;23(6):579-84.

$5 . \quad$ Lundberg IE, Vencovsky J, Alexanderson H. Therapy of myositis: biological and physical. Curr Opin Rheumatol. 2014;26(6):704-11.

6. Alexanderson H, Stenstrom CH, Lundberg I. Safety of a home exercise programme in patients with polymyositis and dermatomyositis: a pilot study. Rheumatology (Oxford). 1999;38(7):608-11.

7. Benatti FB, Pedersen BK. Exercise as an anti-inflammatory therapy for rheumatic diseases-myokine regulation. Nature reviews Rheumatology. 2015;11(2):86-97.

8. $\quad$ Munters LA, Loell I, Ossipova E, Raouf J, Dasmalchi M, Lindroos E, et al. Endurance Exercise Improves Molecular Pathways of Aerobic Metabolism in Patients with Myositis. Arthritis \& Rheumatology. 2016; In press.

9. Munters LA, Dastmalchi M, Andgren V, Emilson C, Bergegard J, Regardt M, et al. Improvement in Health and Possible Reduction in Disease Activity Using Endurance Exercise in Patients With Established Polymyositis and Dermatomyositis: A Multicenter Randomized Controlled Trial With a 1-Year Open Extension Followup. Arthrit Care Res. 2013;65(12):1959-68.

10. Munters LA, Dastmalchi M, Katz A, Esbjornsson M, Loell I, Hanna B, et al. Improved exercise performance and increased aerobic capacity after endurance training of patients with stable polymyositis and dermatomyositis. Arthritis Research \& Therapy. 2013;15(4).

11. Powers SK, Ji LL, Kavazis AN, Jackson MJ. Reactive oxygen species: impact on skeletal muscle. Comprehensive Physiology. 2011;1(2):941-69.

12. Vasilaki A, McArdle F, Iwanejko LM, McArdle A. Adaptive responses of mouse skeletal muscle to contractile activity: The effect of age. Mech Ageing Dev. 2006;127(11):830-9.

13. Gibson JN, Smith K, Rennie MJ. Prevention of disuse muscle atrophy by means of electrical stimulation: maintenance of protein synthesis. Lancet. 1988;2(8614):767-70.

14. Hepple RT, Mackinnon SL, Thomas SG, Goodman JM, Plyley MJ. Quantitating the capillary supply and the response to resistance training in older men. Pflugers Arch. 1997;433(3):238-44.

15. Ostrowski K, Rohde T, Zacho M, Asp S, Pedersen BK. Evidence that interleukin-6 is produced in human skeletal muscle during prolonged running. J Physiol. 1998;508 ( Pt 3):949-53

\section{John Wiley \& Sons}

This article is protected by copyright. All rights reserved. 